Noname manuscript No.

(will be inserted by the editor)

\title{
Solid-state laser system for laser cooling of Sodium
}

\author{
Emmanuel Mimoun ${ }^{1}$, Luigi de Sarlo ${ }^{1}$, Jean-Jacques Zondy ${ }^{2}$, Jean Dalibard ${ }^{1}$, Fabrice Gerbier ${ }^{1}$ \\ 1 Laboratoire Kastler Brossel, ENS, UPMC, CNRS, 24 rue Lhomond, 75005 Paris, France. \\ 2 INM-CNAM, 61 rue du Landy, 93210 La Plaine Saint Denis, France.
}

The date of receipt and acceptance will be inserted by the editor

\begin{abstract}
We demonstrate a frequency-stabilized, allsolid laser source at $589 \mathrm{~nm}$ with up to $800 \mathrm{~mW}$ output power. The laser relies on sum-frequency generation from two laser sources at $1064 \mathrm{~nm}$ and $1319 \mathrm{~nm}$ through a PPKTP crystal in a doubly-resonant cavity. We obtain conversion efficiency as high as $2 \mathrm{~W} / \mathrm{W}^{2}$ after careful optimization of the cavity parameters. The output wavelength is tunable over $60 \mathrm{GHz}$, which is sufficient to lock on the Sodium $\mathrm{D}_{2}$ line. The robustness, beam quality, spectral narrowness and tunability of our source make it an alternative to dye lasers for atomic physics experiments with Sodium atoms.
\end{abstract}

Key words 37.10.De - 42.65.Ky

\section{Introduction}

Among the many atomic species that can be brought to quantum degeneracy, Sodium benefits from low inelastic losses and a relatively large elastic cross-section, allowing the production of large $\left(>10^{8}\right.$ atoms) Bose-Einstein condensates $[1,2,3,4,5,6,7$, or degenerate Fermi clouds by thermalization with the Sodium gas [8]. A current drawback of using Sodium is the necessity of using dye lasers to reach the resonant wavelength of $589.158 \mathrm{~nm}$ (Sodium $\mathrm{D}_{2}$ transition). Although technically well-mastered, dye laser systems are expensive, hardly transportable and comparatively difficult to maintain and operate, justifying the need for alternatives as solid-state lasers.

In addition, new laser sources in the yellow spectral region find applications outside the domain of laser cooling. In fact, the generation of Sodium resonant radiation has been mainly driven by the astronomy community, with the development of high-power $589 \mathrm{~nm}$ lasers to create artificial "beacon" stars by exciting the mesospheric sodium layer [9, 10, 11, 12, 13, 14, 15, 16, 17]. Other possible applications for lasers in the yellow spectral region include Laser-induced detection in the atmospheric range (LIDAR) [18, eye surgery or dermatology [13].

In the literature, several methods for generating continuouswave (cw) laser light around $589 \mathrm{~nm}$ have been reported, including sum-frequency mixing of two infrared lasers around $1319 \mathrm{~nm}$ and $1064 \mathrm{~nm}$ 9, 10, 11, 12, 13, 19, frequencydoubling of a Raman fiber laser [14,15, 16], or sum-frequency mixing of two fiber lasers around $938 \mathrm{~nm}$ and $1535 \mathrm{~nm}$ [17. Applications to laser cooling typically require powers of several hundred $\mathrm{mW}$ to $1 \mathrm{~W}$, the possibility to tune the laser to the Sodium resonance, and a linewidth much smaller than the $\Gamma=2 \pi \times 9.8 \mathrm{MHz}$ natural linewidth of the cooling line.

In a recent paper, we have reported on the realization of a laser source suitable for laser cooling of Sodium [20]. In the present article, we present an exhaustive account of our experimental approach. Our laser source is based on sum frequency generation (SFG) from $1064 \mathrm{~nm}$ and $1319 \mathrm{~nm}$ lasers. SFG is a second order non-linear optical process, in which two pump beams with frequencies $\omega_{1}$ $\left(\lambda_{1}=1064 \mathrm{~nm}\right)$ and $\omega_{2}\left(\lambda_{2}=1319 \mathrm{~nm}\right)$ produce a signal beam with frequency $\omega_{3}=\omega_{1}+\omega_{2}$. We implement this sum frequency technique using commercial, solidstate infrared lasers. The $1064 \mathrm{~nm}$ and $1319 \mathrm{~nm}$ sources are monolithic solid-state lasers built upon an Yttrium Aluminium Garnet (YAG) Non-Planar Ring Oscillator (NPRO) crystal. We also tested another configuration in which the $1064 \mathrm{~nm}$ laser is replaced by an external cavity laser diode boosted by a single-mode fiber amplifier. This led to poorer performances attributed to misbehaviour of the amplifier, and this configuration was not pursued further in our work. The non-linear medium used is a periodically poled potassium titanyl phosphate crystal (pp-KTiOPO $\mathrm{P}_{4}$ or PPKTP), with a poling period chosen to achieve first-order quasi-phase matching (QPM) near room temperature [21,22]. In single-pass configuration, the conversion efficiency is still too small to reach the output power required for laser cooling. To circumvent this problem, the crystal is enclosed in a doubly-resonant 
Emmanuel Mimoun et al.

build-up cavity to enhance the conversion efficiency. Doing so, we reach an overall power conversion efficiency $\alpha_{\text {cav }} \approx 2 \mathrm{~W} / \mathrm{W}^{2}$, where the conversion efficiency is defined through $P_{3}=\alpha P_{1} P_{2}$, with $P_{1,2,3}$ the power at each wavelength. This is to be compared to the value for single-pass conversion efficiency, $\alpha_{\mathrm{sp}} \approx 0.022 \mathrm{~W} / \mathrm{W}^{2}$. In terms of photon fluxes, about $92 \%$ of the photons of the weakest source which enter the cavity are converted [20].

The article is organized as follows. Section 2 gives an overview of our experimental setup. Section 3 recalls the main features of the process of sum frequency generation, and presents our results in a simple single-pass configuration. Section 4 discusses the experimental realization of a doubly resonant cavity and its optimization to achieve near-unit conversion efficiency. Section [5]characterizes the main properties of the laser source obtained at $589 \mathrm{~nm}$.

\section{Experimental setup}

Our experimental setup is represented in Fig. 1. The pump laser sources at $1064 \mathrm{~nm}$ and $1319 \mathrm{~nm}$ are nonplanar ring oscillator YAG lasers (Innolight GmbH, Germany) with an instantaneous spectral linewidth narrower than $10 \mathrm{kHz}$ and output power of $1.1 \mathrm{~W}$ and $500 \mathrm{~mW}$, respectively. Both lasers are collimated to a $1 / e^{2}$ radius around $1 \mathrm{~mm}$ and combined on a dichroic mirror. After passing through an electro-optical phase modulator (EOM) operating near $f_{\bmod } \approx 1 \mathrm{MHz}$, the beams are focused to match their spatial profiles with the fundamental spatial mode of the resonant cavity.

The cavity is built in a bow-tie planar configuration, with highly reflecting mirrors $M_{2}, M_{3}, M_{4}$ and an input coupler $M_{1}$ with lower reflectivities chosen such as to optimize the intracavity conversion (see section 4 below). Mirrors $M_{1}, M_{2}$ are plane, while $M_{3}, M_{4}$ are concave with radius of curvature $R_{c}=100 \mathrm{~mm}$. At the crystal location, the $1064 \mathrm{~nm}(1319 \mathrm{~nm})$ beam is focused to a waist $w_{1}=45 \mu \mathrm{m}\left(w_{2}=47 \mu \mathrm{m}\right)$. This corresponds to almost equal Rayleigh lengths in the crystal $z_{R, i}=\pi n_{i} w_{i}^{2} / \lambda_{i} \simeq 10 \mathrm{~mm}$, with the refractive indices $n_{1} \simeq 1.83$ and $n_{2} \simeq 1.82$ for PPKTP. For our configuration, this choice offers a good trade-off between increasing the nonlinear conversion efficiency and avoiding detrimental effects such as thermal lensing. The cavity geometry is chosen to avoid transverse mode degeneracies, allowing to excite the fundamental Gaussian mode only and suppress higher order modes.

The PPKTP crystal used for SF mixing was manufactured at the Royal Institute of Technology of Stockholm (KTH). Its length is $L=20 \mathrm{~mm}$, with a poling period $\Lambda=12.36 \mu \mathrm{m}$. The use of a periodically poled crystal allows to reach QPM conditions only with temperature tuning (see section 3.1). The crystal is mounted in a copper case with a Peltier thermo-electric cooler element. The case temperature is controlled by a standard
Proportional-Integral-Derivative regulator with better than $10 \mathrm{mK}$ stability. Using the phase-matching curve calculated in section 3.1 we estimate that this corresponds to output power drifts less than $1 \%$.

\section{Single-pass measurements}

In this section, we discuss first our measurements in a single-pass configuration, i.e. without enhancement cavity. As shown later, this measurement is critical to optimize the resonant cavity parameters to reach maximal conversion efficiency. We first recall for completeness the theoretical results relevant to such measurements, first in the simple case where the pumps are plane waves and then in the more realistic situation where they are Gaussian beams. We then discuss our measurements - from which we derive a nonlinear coefficient $\mathrm{d}_{33} \simeq 16 \mathrm{pm} / \mathrm{V}$ for PPKTP.

\subsection{Plane wave model}

In this section, we recall the basic features of SFG using a simplified theoretical framework 22]. The starting point to describe the propagation in the nonlinear crystal are Helmholtz equations, including nonlinear polarization terms. We introduce the complex amplitudes $a_{i}$, related to the electric field strengths by

$$
E_{i}=\sqrt{\frac{2 Z_{0} \hbar \omega_{i}}{n_{i}}} f_{i}(\rho) a_{i}(z) e^{i\left(k_{i} z-\omega_{i} t\right)}
$$

and to the powers by $P_{i}=\hbar \omega_{i}\left|a_{i}\right|^{2}$. Here $k_{i}$ is the momentum of a photon with frequency $\omega_{i}$ in a medium with index of refraction $n_{i}, z$ is the direction of propagation of light, $\rho$ is the transverse coordinate, $f_{i}$ denotes the area-normalized transverse mode for each beam, and $Z_{0}=\sqrt{\mu_{0} / \epsilon_{0}}$ is the impedance of vacuum.

As a first approximation, we neglect the spatial profile of the laser beams and set $f_{i}(\rho)=S^{-1 / 2}$, with a cross-section $S$ identical for all beams. The Helmholtz equation for the harmonic wave $a_{3}$ then reads 22 ]

$$
\frac{d a_{3}}{d z}=-i \gamma a_{1} a_{2} e^{-i \Delta k z}
$$

where the non-linear coupling coefficient $\gamma$ can be written as

$$
\gamma=\left(\frac{2 \hbar \omega_{1} \omega_{2} \omega_{3} Z_{0}^{3} \epsilon_{0}^{2} d^{2}}{S n_{1} n_{2} n_{3}}\right)^{1 / 2}
$$

Here $d$ denotes the nonlinear coefficient which characterizes the efficiency of the nonlinear process 1 , and $\Delta k=$ $k_{3}-k_{1}-k_{2}$ is the phase mismatch parameter. In a bulk

1 We assume here that the laser polarizations are parallel and aligned with the principal axis of the non-linear medium characterized by the largest non-linear coefficient $d_{33}$. 


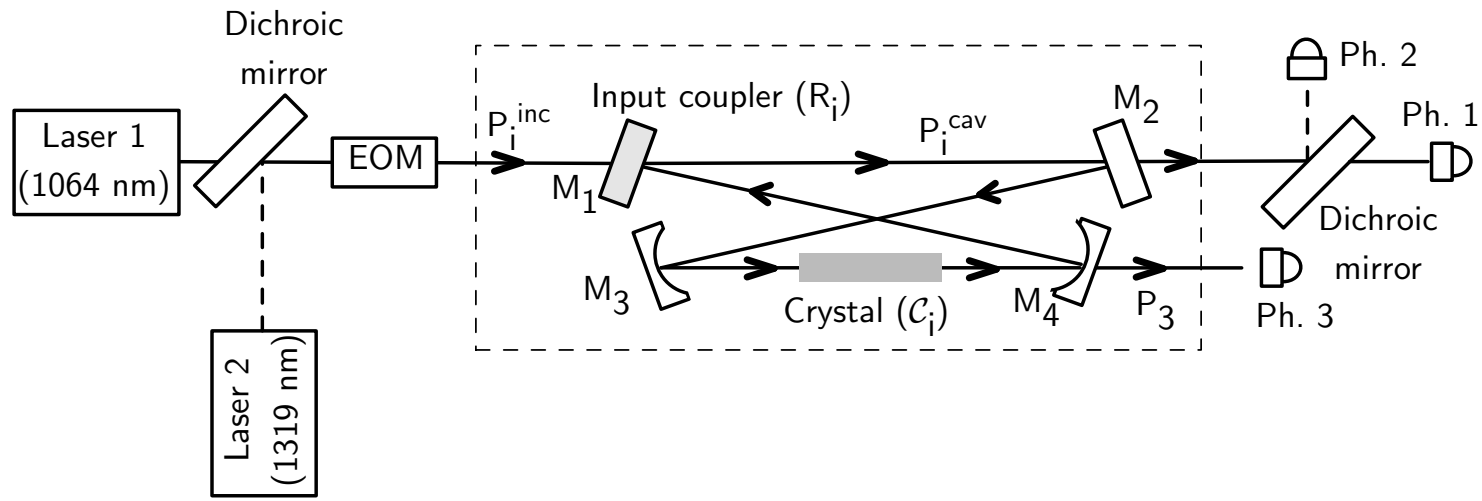

Figure 1 Overview of the laser system. Mirrors M1 (with reflectance $\mathrm{R}_{i}$ at wavelength $\lambda_{i}$ ) and M2 are flat, while mirrors M3 and M4 are concave with a radius of curvature of $10 \mathrm{~cm}$ to allow focusing in the crystal, in which a fraction $\mathcal{C}_{i}$ of the power at wavelength $\lambda_{i}$ is converted. The incoming power at wavelength $\lambda_{i}$ is noted $P_{i}^{\text {inc }}$, and the intra-cavity power $P_{i}^{\text {cav }}$. Ph. stands for Photodiode and EOM for Electro-Optic phase Modulator.

crystal, $d$ can be treated as constant over the crystal length (neglecting possible defects and impurities). In contrast, a periodically poled crystal is characterized by an alternating permanent ferromagnetization [21. As a result, $d$ is a periodic function of the position $z$ in the poling direction, with spatial period $\Lambda$. As such, it can be expanded as a Fourier series, $d(z)=d_{33} \sum_{n} c_{n} e^{i q_{n} z}$, where $q_{n}=2 \pi n / \Lambda$. Significant conversion only takes place when the QPM condition $q_{n}=\Delta k$ occurs for some integer $n$. Here we only consider the first term of the series, $n=1$ (first order QPM). For a $50 \%$ poling dutycycle, the Fourier coefficient $c_{1}$ is $2 / \pi$ and the nonlinear coefficient $d$ in Eq.(2) becomes an effective coefficient $d_{\mathrm{pp}}=2 / \pi d_{33}$. Hence, the maximum efficiency is lower than for a bulk crystal with perfect phase matching by a factor $4 / \pi^{2} \approx 0.4$.

Assuming low conversion, we solve for $a_{3}$ in the undepleted pumps approximation, $a_{i}(z) \approx a_{i}(0)$, for $i=1,2$. For a crystal of length $L$, this gives the generated power at $589 \mathrm{~nm}$ as

$$
P_{3}=\alpha_{\mathrm{sp}} P_{1} P_{2} \operatorname{sinc}^{2}\left(\frac{\left(\Delta k-\frac{2 \pi}{\Lambda}\right) L}{2}\right),
$$

where $\operatorname{sinc}(x)=\sin (x) / x$ and where the maximal singlepass conversion efficiency $\alpha_{\mathrm{sp}}$ is

$$
\alpha_{\mathrm{sp}}=\frac{\gamma^{2} L^{2}}{\hbar} \frac{\omega_{3}}{\omega_{1} \omega_{2}}
$$

As we will see, the single-pass efficiency $\alpha_{\text {sp }}$ is the critical parameter to allow optimization of the resonant cavity. The argument of the sinc function in Eq.(3) depends on temperature through the various refractive indices (the case of KTP has been studied experimentally in 23 , 24). Therefore, by adjusting the temperature one can reach the quasi-phase matching condition $\Delta k=2 \pi / \Lambda$ which maximizes the conversion efficiency. In practice, the period $\Lambda$ is chosen so that this condition is fulfilled near room temperature.

\subsection{Boyd-Kleinmann theory}

Instead of collimated beams, experiments use focused Gaussian beams in order to reach high intensities, and hence efficient conversion. Non-linear processes with Gaussian waves were studied in a seminal paper by Boyd and Kleinmann [25], where explicit expressions were given for the conversion efficiency in the undepleted pumps approximation (see also [26]). The general expressions are rather complex, but they can be drastically simplified by assuming identical Rayleigh lengths $z_{\mathrm{R}}$ for the three beams. Indeed, both infrared beams are resonant in the cavity, which implies that their confocal parameter is the same, essentially determined by the geometry of the cavity. Although the output beam generated by SFG is not resonant, it is generated only in the regions where both pump beams overlap significantly so that approximating its spatial mode by a Gaussian beam with the same confocal parameter as the infrared ones is a reasonable assumption 22 .

With Gaussian beams, the coefficient $\gamma$ defined in Eq.(2) becomes a function of $z$ proportional to the overlap integral $I(z)=\int d^{(2)} \rho f_{1} f_{2} f_{3}^{*}$ between the different modes $f_{i}$. For Gaussian waves with waists $w_{i}$ at the crystal center, and Rayleigh length $z_{R}=\pi n_{i} w_{i}^{2} / \lambda_{i}$, this can be calculated explicitly. After some rearrangement, the expression for the output power can be writtten as $P_{3}=\alpha_{\mathrm{sp}} P_{1} P_{2}$, where the single-pass conversion efficiency $\alpha_{\mathrm{sp}}$ reads

$$
\alpha_{\mathrm{sp}}=Z_{1} \frac{d_{\mathrm{pp}}^{2} L}{\lambda_{3}^{3}} h(a, b, c)
$$

Here

$$
Z_{1}=\frac{32 \pi Z_{0}}{\lambda_{1} \lambda_{2}\left(\frac{n_{1}}{\lambda_{1}}+\frac{n_{2}}{\lambda_{2}}+\frac{n_{3}}{\lambda_{3}}\right)^{2}} \approx 2.15 \mathrm{k} \Omega
$$


has the dimension of an impedance and the dimensionless function $h$

$$
h(a, b, c)=\frac{1}{4 a}\left|\int_{-a}^{a} \frac{e^{i b \tau}}{(1+i \tau)(1+i c \tau)} d \tau\right|^{2}
$$

is the so-called Boyd-Kleinman factor. The latter depends on the reduced variables $a=\frac{L}{2 z_{R}}, b=\left(\Delta k-\frac{2 \pi}{\Lambda}\right) z_{R}$, and $c=\Delta k w_{\text {eff }}^{2} / z_{R}$, with $w_{\text {eff }}^{-2}=\left(\pi / z_{R}\right) \sum_{i} n_{i} / \lambda_{i}$. We can further write $c=\frac{\Delta k w_{\text {eff }}^{2}}{z_{R}}=\left(b+\frac{1}{a} \frac{\pi L}{\Lambda}\right) \times \frac{w_{\text {eff }}^{2}}{z_{R} L} \times a$, showing that the function $h$ depends only on the variables $a$ and $b$ once the wavelengths, crystal length and crystal period are fixed 2 . Since $\frac{w_{\text {eff }}^{2}}{z_{R} L} \sim \frac{\lambda_{3}}{2 \pi n_{3} L} \ll 1, c=0$ can be assumed, and the integral $h(a, b, c)$ is well approximated by

$$
h(a, b, 0)=\frac{1}{4 a}\left|\int_{-a}^{a} \frac{e^{i b \tau}}{1+i \tau} d \tau\right|^{2} .
$$

There are two limiting cases of interest :

1. Collimated beams, $z_{R} \gg L$ or $a \ll 1$ : in this case we find the sinc function familiar from the plane wave case (see Eq.(3) ),

$$
h(a, b, 0) \approx a \operatorname{sinc}^{2}((b+1) a) .
$$

2. Focused beams, $z_{R} \ll L$ or $a \gg 1$ : for tightly focused beams, the length $L$ of the crystal naturally drops out of the problem. One finds that $h$ tends to a limit function

$$
h(a \gg 1, b, 0) \approx \begin{cases}\frac{\pi^{2}}{a} e^{-2 b}, & b>0, \\ 0, & b<0 .\end{cases}
$$

The experimental procedure of changing the temperature (which changes $\Delta k$ ) corresponds to searching for the maximum $h^{*}(a)$ of $h(a, b, 0)$ as a function of $b$ for a fixed $a$ [25]. The optimum phase mismatch is offset from the plane wave result $(\Delta k=2 \pi / \Lambda)$ by a quantity on the order of $z_{R}^{-1}$, a consequence of the Gouy phase accumulated as the beams pass through a focus in the crystal. The optimal focusing corresponds to the maximum of $h^{*}$, which is found for $a^{*}=L / 2 z_{R} \approx 2.84$ ( $\left.h^{*}(2.84) \approx 1.06\right)$. This optimum is quite loose, as $h^{*}>1$ for $1.5 \lesssim a \lesssim 5$.

\subsection{Results for single pass operation}

Our experimental configuration corresponds to a configuration where $z_{R} \approx 10 \mathrm{~mm}$, or $a \approx 2$ for a $20 \mathrm{~mm}$ long crystal. For this parameter, the shape of the function $h$ is very close to the sinc function predicted by

\footnotetext{
${ }^{2}$ In principle, $Z_{0}$ and $c$ depend weakly on temperature as $\Delta k$ through the dependance of the indices. One finds that over a temperature range of $20^{\circ}-100^{\circ}$ the relative variations do not exceed a few $10^{-4}$. Thus, we can safely consider $Z_{0}$ and $c$ as constants for the rest of the calculations.
}

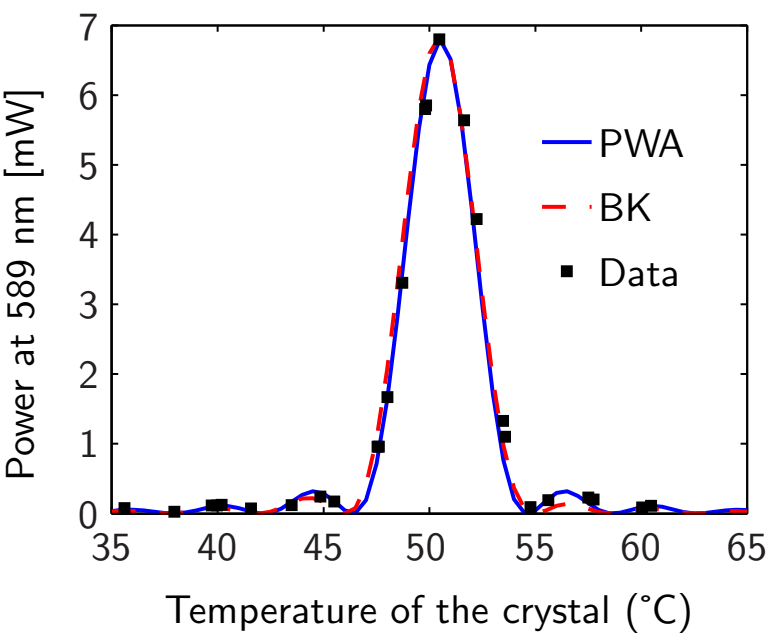

Figure 2 Power produced at $589 \mathrm{~nm}$ in a single-pass configuration when the temperature of the PPKTP crystal is varied. The plot is fitted for a value of the poling period $\Lambda=12.32 \mu \mathrm{m}$, for the Plane Wave Approximation (solid line, PWA) and the Boyd Kleinmann theory (dashed line, BK). The peak value measured for the power produced at the output of the cavity is $7 \mathrm{~mW}$. Considering the losses due the optics used to separate yellow light from infrared, we infer a total produced power of $8.5 \mathrm{~mW}$.

the plane wave model (see Fig. 21). To perform singlepass measurements, we use the same setup as in Fig. 1 but remove the mirror $M_{4}$ from the cavity. We find that the optimum temperature $T_{\mathrm{QPM}}^{(\mathrm{mes})} \approx 50^{\circ} \mathrm{C}$ for our crystal samples. For this temperature, the refractive indices are $n_{1} \approx 1.83, n_{2} \approx 1.82$, and $n_{3} \approx 1.87$. Using BK theory to fit the data, we measure an efficiency $\alpha_{\mathrm{sp}}^{\mathrm{mes}} \approx 0.022 \mathrm{~W}^{-1}\left(P_{3}=9 \mathrm{~mW}\right)$, obtained for normal incidence on the crystal using $P_{2}=440 \mathrm{~mW}$ and $P_{1}=940 \mathrm{~mW}$. The variation of the output power with the power of both infrared lasers are found to be linear, confirming the validity of the undepleted pumps approximation for a single pass operation. Boyd-Kleinmann theory predicts a value $\alpha_{\mathrm{sp}}^{\mathrm{BK}} \approx 0.021 \mathrm{~W} / \mathrm{W}^{2}$ for $d_{33} \approx$ $16 \mathrm{pm} / \mathrm{V}$, which is in a good agreement with values for the non-linear coefficient found in the literature [27,28, 29. Note also that this is quite close to the optimal value $\alpha^{*} \approx 0.023 \mathrm{~W} / \mathrm{W}^{2}$ which would be obtained for slightly tighter focusing. Applying the equations (3+4) obtained in the plane wave approximation, one would expect $\alpha \approx 0.022$, taking for the cross section $S$ the average of the waist of a gaussian beam over the length $\mathrm{L}$ of the crystal : $S=\frac{1}{L} \int_{-L / 2}^{L / 2} \pi w^{2}(z) / 2 d z$, with $w(0)=45 \mu \mathrm{m}$. This highlights the usefulness of these theories for quantitative predictions.

The PPKTP crystals used in our experiments was ordered with a poling period $\Lambda=12.36 \mu \mathrm{m}$. Using the val-

3 This is the highest value obtained so far and differs from the measurements presented in Fig. 2. which we have taken with another laser source at $1064 \mathrm{~nm}$ of poorer quality, and another crystal. 
ues given in Refs. 23, 24 for the temperature and wavelength dependence of the refractive indices, we calculate a $\mathrm{QPM}$ temperature $T_{\mathrm{QPM}}^{(\text {calc }} \approx 28^{\circ} \mathrm{C}$, apparently far from the measured $T_{\mathrm{QPM}}^{(\mathrm{mes})} \approx 50^{\circ} \mathrm{C}$ (see Fig. 2). We note however that the quasi-phase-matching temperature is rather sensitive to the exact value of the period. Using the same wavelength and temperature dependence for the refraction indices, we find that the measured $T_{\mathrm{QPM}}^{(\mathrm{mes})}$ corresponds to a spatial period $\Lambda=12.32 \mu \mathrm{m}$.

\section{Intra-cavity conversion}

\subsection{Definition of the optimization problem}

After having characterized the single-pass sum-frequency process, we turn to the cavity setup. The presence of the cavity enhances the infrared lasers intensities at the crystal location. For the geometry shown in Fig. 11 the intracavity power at resonance can be written for each infrared laser as 30.

$$
P_{i}^{\mathrm{cav}}=\frac{T_{i}}{\left(1-\sqrt{R_{i} \cdot\left(1-\delta_{i}\right) \cdot C_{i}}\right)^{2}} P_{i}^{\mathrm{inc}}, \quad i=1,2
$$

In Eq. (10), $P_{i}^{\text {cav }}$ denotes the circulating intracavity power, $P_{i}^{\text {inc }}$ the incident power coupled into the fundamental mode of the cavity, $R_{i}, T_{i}$ denote the input coupler (mirror $\left.M_{1}\right)$ reflectance and transmittance $\left(R_{i}+T_{i}+L_{i}=1\right.$, with $L_{i}$ a loss coefficient), $\delta_{i}$ denote the passive losses after one round trip, excluding the input coupler (i.e. finite reflectances of the other mirrors, and losses in the crystal), and $C_{i}$ accounts for the nonlinear conversion. To evaluate $C_{i}$, we use conservation laws for the photon fluxes which state that $\left|a_{1}\right|^{2}+\left|a_{3}\right|^{2}$ and $\left|a_{2}\right|^{2}+\left|a_{3}\right|^{2}$ are constant along the crystal length (in the absence of absorption). This corresponds to non-linear conversion factors given by

$$
C_{i}=1-\frac{\lambda_{3} P_{3}}{\lambda_{j} P_{j}^{\text {cav }}}, \quad(j \neq i ; i, j=1,2) .
$$

Assuming total transmission of the yellow light by the output mirror M4, the $589 \mathrm{~nm}$ power $P_{3}$ coupled out of the cavity is given by $P_{3} \approx \alpha P_{1}^{\text {cav }} P_{2}^{\text {cav }}$, under the undepleted pump approximation. When both pumps have imbalanced powers, the output power at $\omega_{3}$ is ultimately limited by the weakest one, since one photon from both pumps is required to create one at $\omega_{3}$. As it is the case in our experiment, we assume that the weakest source is the one at wavelength $\lambda_{2}$. This translates into a maximum power $P_{3}^{\max }=\left(\lambda_{2} / \lambda_{3}\right) P_{2}^{\text {inc }}$. Therefore, a figure of merit to characterize the conversion efficiency is the ratio

$$
\eta=\frac{P_{3}}{P_{3}^{\max }}=\frac{\lambda_{2}}{\lambda_{3}} \frac{P_{3}}{P_{2}^{\text {inc }}}
$$

between the actual power and the absolute maximum power that can be obtained from the available pump power coupled into the cavity $P_{2}^{\text {inc }}$.

The problem at hand is thus to maximize $\eta$ for given cavity parameters $\delta_{i}, L_{i}, \alpha$. This amounts to balancing the input coupler reflectances $R_{1}, R_{2}$ with the total loss per round trip, including the non-linear conversion. This is usually termed impedance matching 31. In our case, finding the impedance matching point is a coupled problem, since one should maximize simultaneously both intensities in the cavity using (10) and (11). This last equation is critically dependent on the single-pass conversion coefficient $\alpha$.

\subsection{Total conversion in an idealized lossless cavity}

Let us first study the case, where passive losses in the cavity and on the input coupler can be neglected $\left(\delta_{i}, L_{i}=\right.$ 0 in the above equations). The question to be answered is whether it is possible to convert all photons at $\lambda_{2}$ into photons at the harmonic at $\lambda_{3}$ (cavity conversion efficiency $\eta=1$ ). In [20], we showed that this is indeed the case for any value of the input coupler reflectance $R_{2}$. We recall here the argument for completeness. We look for a solution where the output flux at $\lambda_{3}$ and the incident flux at $\lambda_{2}$ are equal, $P_{3} /\left(\hbar \omega_{3}\right)=P_{2}^{\text {inc }} /\left(\hbar \omega_{2}\right)$. According to Eq.(11), this corresponds to $\mathcal{C}_{2}=1-\frac{P_{2}^{\text {inc }}}{P_{2}^{\text {cav }}}$. The cavity equation (10) becomes

$$
P_{2}^{\text {cav }}=\frac{\left(1-R_{2}\right) P_{2}^{\text {inc }}}{\left(1-\sqrt{R_{2}\left(1-\frac{P_{2}^{\text {inc }}}{P_{2}^{\text {cav }}}\right)}\right)^{2}}
$$

This solves into the simple result

$$
P_{2}^{\text {cav }}=\frac{P_{2}^{\text {inc }}}{1-R_{2}}
$$

valid for any $R_{2}$. Thus we conclude that there is always a possibility to reach complete conversion in the ideal, lossless case, corresponding to the intracavity flux for the weak pump 2 as given above. The flux for the strong pump 1 is found from the relation $P_{3}=\alpha P_{1}^{\text {cav }} P_{2}^{\text {cav }}$,

$$
P_{1}^{\mathrm{cav}}=\frac{\left(1-R_{2}\right) \lambda_{2}}{\alpha_{\mathrm{sp}} \lambda_{3}}
$$

The parameters of the cavity $\left(R_{1}, R_{2}\right)$ are linked via Eq.(10). For any $R_{2}$, one can find a value of $R_{1}$ leading to the power $P_{1}^{\text {cav }}$ given above, corresponding to complete conversion of the $\lambda_{2}$ photons.

\subsection{Optimization of conversion for a realistic lossy cavity}

In any practical situation, passive losses will be present. This modifies the conclusions of the last subsection, as 
these losses limit the enhancement factor that can be reached in the cavity. Unlike the lossless case, instead of a locus of optimal points in the $\left(R_{1}, R_{2}\right)$ plane, one finds a unique value of $\left(R_{1}, R_{2}\right)$ that maximizes $P_{3}$, at a value smaller than $P_{3}^{\max }$. However, this optimum is quite loose when non-linear conversion dominates over the passive losses $\left(\mathcal{C}_{i} \gg \delta_{i}\right)$. This highlights the importance of a large single-pass efficiency, justifying the use of a highly nonlinear material such as PPKTP : the required power $P_{1}$ is reduced (see Eq.(15)), making the cavity more tolerant to passive losses.

We rewrite $\mathcal{C}_{2}=1-\eta \frac{P_{2}^{\text {inc }}}{P_{2}^{\text {cav }}}$. The cavity equation for wave 2 then leads to two solutions for the intracavity power

$P_{2}^{\text {cav }}=\frac{(1+r) T_{2}-(1-r) r \eta}{(1-r)^{2}} \pm 2 \frac{\sqrt{T_{2} r\left(T_{2}-(1-r) \eta\right)}}{(1-r)^{2}}$

with $r=R_{2}\left(1-\delta_{2}\right)$ the total passive loss coefficient for the circulating waves. The existence of two solutions indicates a possible bistability. Such solutions are real provided

$$
T_{2} \geq(1-r) \eta
$$

When this condition is not fulfilled, the cavity is unstable due to excessive passive or nonlinear losses. This condition sets a limit on the efficiency achievable for given cavity parameters $T_{2}, R_{2}, \delta_{2}, \eta<\eta^{\max }=T_{2} /(1-r)$. Assuming one chooses the input coupler to reach this maximum value, one finds for small losses $\left(\delta_{2}, L_{2} \ll R_{2}\right)$ an intracavity power

$$
P_{2}^{\mathrm{cav}} \approx \frac{P_{2}^{\mathrm{inc}}}{1-R_{2}}\left(1-\frac{L_{2}+2 R_{2} \delta_{2}}{1-R_{2}}\right),
$$

close to the idealized case studied before.

Table 1 Reflectances and transmittances of the optical elements inside the cavity, at both wavelengths. Reflectances are measured within $0.5 \%$ and transmittances within $0.2 \%$. The values for the crystal are specifications by the manufacturer. $\mathcal{R}$ is the optimum given by the numerical simulation.

\begin{tabular}{r|ccc|ccc}
\hline \hline & \multicolumn{3}{|c|}{$1064 \mathrm{~nm}$} & \multicolumn{3}{c}{$1319 \mathrm{~nm}$} \\
& $\mathrm{R}$ & $\mathcal{R}$ & $\mathrm{T}$ & $\mathrm{R}$ & $\mathcal{R}$ & $\mathrm{T}$ \\
\hline$M_{1}$ & 0.930 & 0.96 & 0.060 & 0.740 & 0.79 & 0.250 \\
$M_{2}, M_{3}, M_{4}$ & 0.995 & & 0.005 & 0.995 & & 0.005 \\
Crystal & & & 0.980 & & & 0.980
\end{tabular}

The solution of the coupled equations giving $P_{1}^{\text {cav }}, P_{2}^{\text {cav }}$ [Eq.(10)] is performed numerically 4 , using as input the

\footnotetext{
4 A numerical algorithm maximizing the two dimensional function $P_{1}^{\text {cav }} P_{2}^{\text {cav }}=f\left(R_{1}, R_{2}\right)$ was implemented. In practice, $P_{1}^{\text {cav }}$ is first evaluated using an Euler secant method by substituting $P_{2}^{\text {cav }}$ in $C_{1}$ [Eq. [11]] by its expression given by Eq. (10). Once $P_{1}^{\text {cav }}$ solved, its value is used to derive $P_{2}^{\text {cav }}$
}

available power in our infrared sources and the measured characteristics of the cavity. We carried out the optimization with respect to the input coupler transmittances at both infrared wavelengths [20].

Experimentally, we characterized carefully the transmition and reflection coefficients of the mirrors used for the cavity (see Table 1). The measured reflectances correspond to a passive (i.e. without non-linear conversion) amplification of the intra-cavity power by a factor around 22 at $1064 \mathrm{~nm}$ and 12 at $1319 \mathrm{~nm}$. To find the powers coupled into the cavity fundamental mode, we sent the lasers independently into the cavity with a known incident power. Comparing the measured intracavity power (inferred from the power transmitted through M2 and the measured value of its transmittance) with the one expected from the reflectances gives the fraction of incident power effectively coupled to the fundamental mode, around $85 \%$ for both wavelengths. The measured reflectances as well as coupling efficiencies were taken into account in our numerical simulations, predicting a conversion efficiency $\eta \simeq 0.9$ for the photons at $\lambda_{2}$ coupled into the cavity (see [20]). The maximal measured output power of $800 \mathrm{~mW}$, which corresponds to $\eta=0.92$, is in fair agreement with this result.

\subsection{Cavity setup and locking}

An essential requirement to achieve a stable output with high efficiency is to ensure that both pump lasers are simultaneously resonant in the cavity. In order to maintain the cavity on resonance for both wavelengths, a double locking scheme using the stable $1319 \mathrm{~nm}$ source as a master laser is implemented (see Fig. 3a). Both lasers are routed together through an electro-optical modulator (EOM) placed before the cavity, and resonantly driven at a frequency $f_{\text {mod }}=1 \mathrm{MHz}$. This dithers the laser frequencies and generate a dispersive signal from the cavity transmission. In our implementation, the weaker pump laser 2 is used as a master laser onto which the cavity length is locked using an integrating servo-loop. In a second step, the stronger pump 1 laser is locked onto the cavity, and consequently on the master laser, ensuring stable operation of the ensemble. In details, the small fraction of infrared light transmitted by the second mirror $\mathrm{M}_{2}$ is collected by two separate photodiodes (see Fig. 1). Two piezoelectric transducers glued to the cavity mirrors $\mathrm{M}_{2}$ and $\mathrm{M}_{3}$ are used to tune the cavity length. The first one $\left(\mathrm{M}_{3}\right)$ allows fast response in the $30 \mathrm{kHz}$ range, but has a limited travel of a few tens of nm. The second piezoelectric stack allows to correct for larger drifts of the cavity length, occuring over much longer timescales (from a few ms to a few hours). The

and the function $f$. The optimal couple $\left(R_{1}^{\text {opt }}, R_{2}^{\text {opt }}\right)$ is then tracked using an adaptative stepsize algorithm maximizing $f$. 

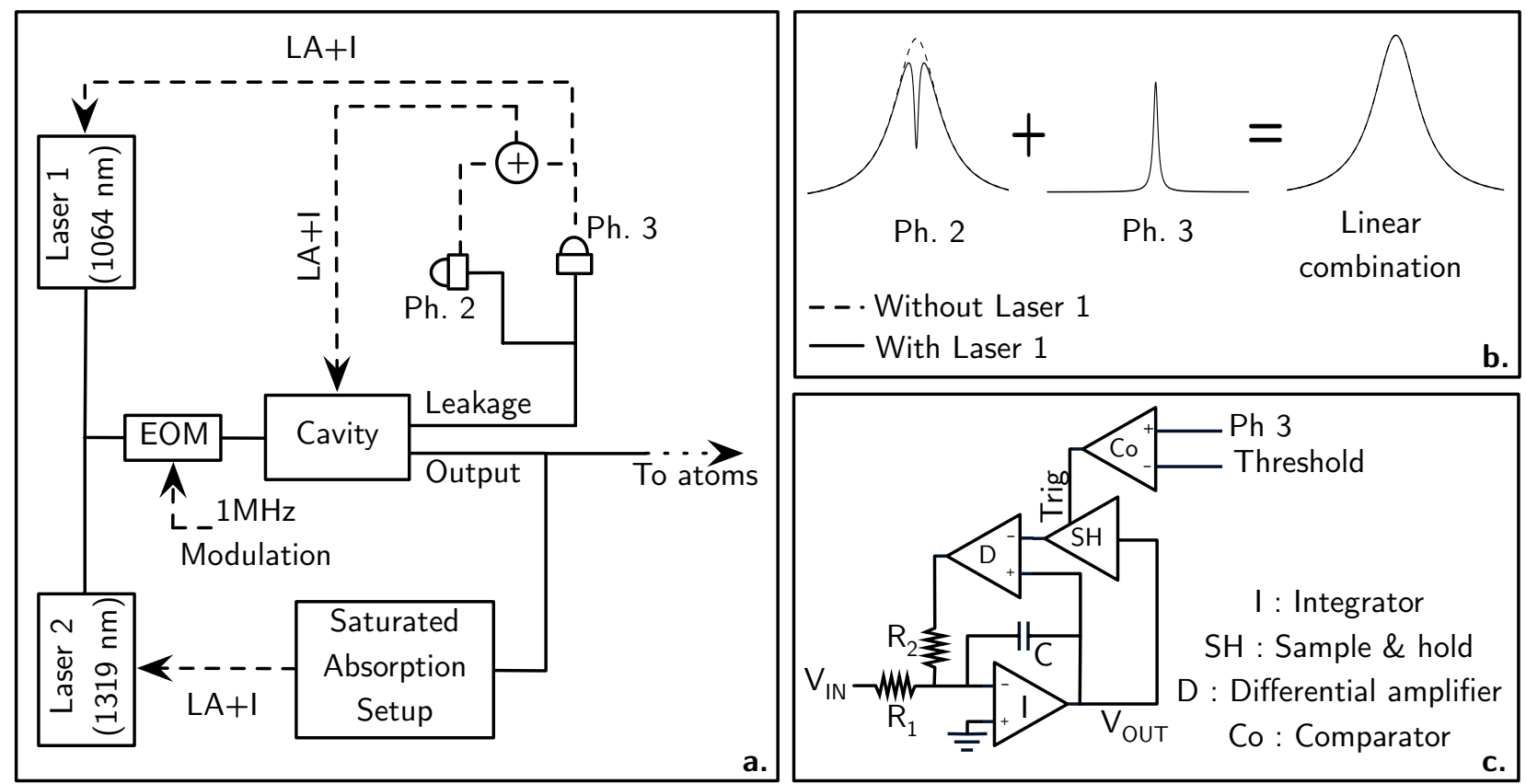

Figure 3 a: Locking scheme for the SFG cavity. Solid lines represent optical paths, while dashed lines indicate electronic connections. LA + I: Use of a lock-in amplifier and an integrator to lock the lasers or the cavity to the maximum of a signal produced by one of the photodiode (Ph.). b: Linear combination used to compensate for the dip in intra-cavity power due to conversion. The photodiode monitoring laser 2 sees a power drop while the photodiode monitoring laser 3 sees a peak. Summing them up allows one to always maintain a peak signal to lock to. c: Automatic gain control circuit to control integrator saturation. COMP: comparator, SH: sample-and-hold amplifier, INT: integrator, D: precision differential amplifier.

photodiode signal at $1319 \mathrm{~nm}(\mathrm{Ph} .2)$ is demodulated by a lock-in amplifier operating at the modulation frequency $f_{\text {mod }}$ driving the EOM, producing a dispersive error signal subsequently fed back to both piezoelectric transducers (with appropriate filters in the feedback loop). This locks the length of the cavity on the stable $1319 \mathrm{~nm}$ source. The photodiode signal at $589 \mathrm{~nm}$ (Ph.3) is demodulated in a similar way, and the resulting signal can be used to react on laser 1 frequency using available piezoelectric and temperature control to ensure that it follows the cavity resonance.

In situations where the conversion efficiency is large, this standard locking scheme leads to serious stability problems with both IR lasers simultaneously present in the cavity. To see this, picture a situation where the cavity is on lock with the 1064 laser off-resonant. As the $1064 \mathrm{~nm}$ laser frequency is tuned to reach resonance, the power level of the $1319 \mathrm{~nm}$ drops due to conversion into $589 \mathrm{~nm}$ photons (see Fig. $3 \mathbf{b}$ ). This large drop of the $1319 \mathrm{~nm}$ power level when both lasers resonate cannot be distinguished from a perturbation by the cavity lock. Hence, the cavity lock actually works against keeping both lasers on resonance simultaneously, and resists increasing the conversion efficiency above the level where the $1319 \mathrm{~nm}$ lineshape is distorted significantly. We have devised a simple solution to this problem [20,32. First, instead of the bare $1319 \mathrm{~nm}$ photodiode transmission, the error signal for the cavity lock is derived from a linear combination of this transmission signal and of the yellow output of the laser. The combination is done electronically before the lock-in amplifier, with weights empirically chosen to minimize distortions of the cavity lineshape and to optimize the servo gain around the lock point. Our "fringe reshaping" method works for any level of conversion, and allows stable operation of the laser on a day time scale, even at the highest efficiencies. Second, choosing the $589 \mathrm{~nm}$ output as the error signal for the second servo-loop instead of the $1064 \mathrm{~nm}$ transmission ensures that the system locks to the maximal converted power. Our method relies on the fact that the SFG is a phase-coherent process : a modulation sideband present on the $1319 \mathrm{~nm}$ laser is automatically present on the output (with a different weight that depends on the $1064 \mathrm{~nm}$ power). Synchronous demodulation by the lock-in amplifier therefore preserves the linear combination.

When these two servos are in action, the cavity is doubly resonant, and the two lasers are frequency locked to each other. We have found that the lock of the second laser was somewhat sensitive to disturbances occurring near the optical table. This is a well-known features of integrating servo-loops, which typically encounter difficulties to recover from disturbances with large amplitude that cause the integrator to saturate [33. Integrators are required to achieve zero DC errors in a servo loop, and replacing them with a simpler proportional control is not an option. We have implemented an electronic circuit that bypasses this problem and prevents the integrator from saturating after violent perturbations, while 
maintaining the laser locked at all times. This can be seen as an automatic gain control circuit that limits the DC gain when the input becomes too large. The circuit, shown schematically in Fig $3 \mathrm{c}$, uses the laser power level to detect such disturbances, and compares it to a preset threshold value (set to $80 \%$ of the nominal value in our case). A sample and hold amplifier ( $\mathrm{SH}$ ) samples the integrator voltage, with its output connected ("bootstrapped") to the integrator input through a differential amplifier. Regular operations correspond to the $\mathrm{SH}$ in "sample" mode, where the output closely tracks the input. The output $V_{\text {diff }}$ of the differential amplifier is zero and the integrator behaves normally. When the output of the laser falls below the threshold, the comparator triggers the SH circuit to switch to "hold" mode. The $\mathrm{SH}$ output is frozen at the value $V_{\text {th }}$ it had at threshold, so that the differential amplifier output becomes $V_{\text {diff }}=V_{\text {out }}-V_{\text {th }}$. The output voltage at frequency $\omega$ then becomes

$$
V_{\text {out }}=-\frac{\frac{R_{2}}{R_{1}} V_{\mathrm{in}}-V_{\mathrm{th}}}{1+i R_{2} C \omega},
$$

where $V_{\text {in }}$ is the incoming error signal and $V_{\text {out }}$ the output of circuit. The integrator is thus neutralized before saturating, and the circuit behaves as a proportional controller around the threshold value. When the perturbation is removed, the SH turns back to sample mode and restores regular integrator operation. We use this circuit on all servo controllers in the laser system.

With this last improvement, the system can withstand severe mechanical perturbations without unlocking and requires very little maintenance compared to dye lasers. It is mostly insensitive to temperature fluctuations because the infrared lasers are thermally stabilized and the cavity length fluctuations are compensated by the servo. Alignement is left untouched over weeks, with a power drop below 10\%, and when needed adjustments are only required on the injection path into the cavity. The cavity alignment itself has not been touched for six months. The laser stays locked for a day on its own, and for several hours when tracking an atomic line.

Finally, the frequency drift of the yellow laser is cancelled by locking laser 2 to the $\mathrm{D}_{2}$ line of $\mathrm{Na}$ using standard saturated absorption spectroscopy, yielding a longterm frequency-stabilized laser source.

\section{Yellow laser characterization}

\subsection{Beam quality}

We have characterized the spatial mode of the laser emerging from the cavity. The output beam was focused through a converging lens and imaged on a charge-coupled device (CCD) camera at various distances from the lens. The beam profile for each distance was fitted to a gaussian with $1 / e^{2}$ radius $w$ identified as the beam waist

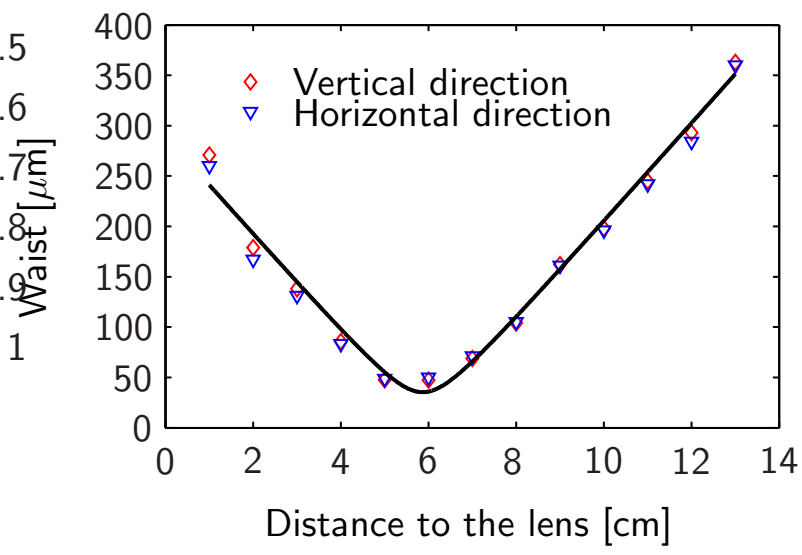

Figure 4 Measurement of the $\mathrm{M}^{2}$ coefficient for the $589 \mathrm{~nm}$ laser in the vertical (triangles) and horizontal (circles) directions. The laser is focused with a $f=100 \mathrm{~mm}$ converging lens at the output of the cavity.

(see Fig. 4). We fitted this function to $w_{0} \sqrt{1+\theta\left(z / w_{0}\right)^{2}}$, where $w_{0}$ is the waist of the beam near focus, $\theta$ is its divergence, and $z$ the direction of propagation. This gives a $\mathrm{M}^{2}$ parameter $\mathrm{M}^{2}=\pi w_{0} \theta / \lambda=1.02$, indicating diffractionlimited performances. This shows the high-quality of the transverse mode of the output beam. Measurements in both transverse directions show no visible astigmatism.

\subsection{Intensity noise measurements}

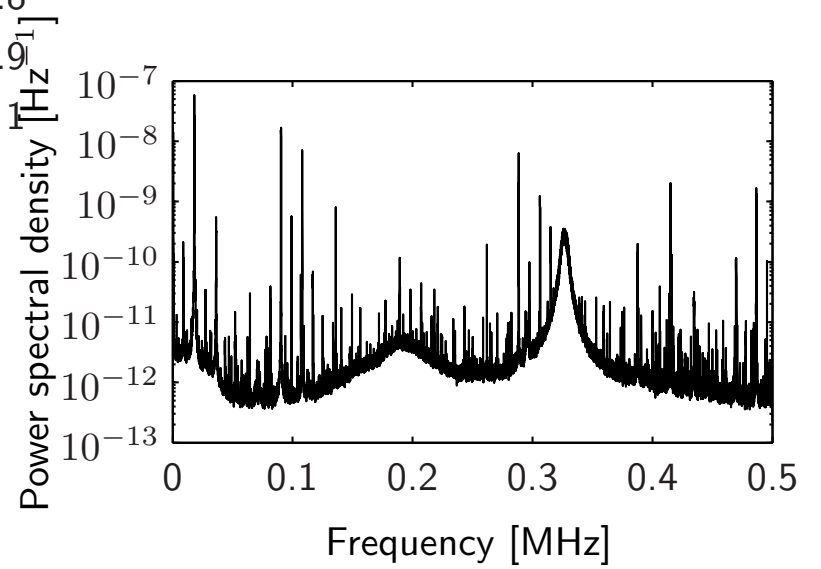

Figure 5 Relative intensity noise spectral density of the laser source averaged over 100 samples.

Intensity noise of the output was characterized by recording the beam power on a fast photodiode (bandwidth $10 \mathrm{MHz}$ ) followed by a 16 bits analog acquisition card (National Instruments NI-6259). From such samples, we determined the one-sided power spectral density $S_{\mathrm{RIN}}$ of the instantaneous intensity normalized to the mean intensity,

$$
S_{\mathrm{RIN}}(\nu)=\frac{1}{T}\left\langle\left|\int_{0}^{T} \frac{I(\tau)}{\langle I\rangle} e^{i 2 \pi \nu \tau} d \tau\right|^{2}\right\rangle,
$$


where $\langle\cdots\rangle$ denotes statistical averaging and where $T \approx$ $100 \mathrm{~ms}$ is the measurement time. The results averaged over 100 samples are shown in Fig. 5. This corresponds to a noise $\delta I /\langle I\rangle \approx 4 \times 10^{-3}$ integrated over a $5 \mathrm{~Hz}-500 \mathrm{kHz}$ bandwidth. Two broad noise peaks are visible near $190 \mathrm{kHz}$ and $330 \mathrm{kHz}$, which probably reflect resonances in the cavity piezoelectric actuators. The noise level is sufficient for our application, but could be controlled actively to a lower level if needed, for instance by monitoring the instantaneous power and reacting on the incident power from the $1064 \mathrm{~nm}$ laser.

\subsection{Absorption from laser-cooled Sodium atoms}

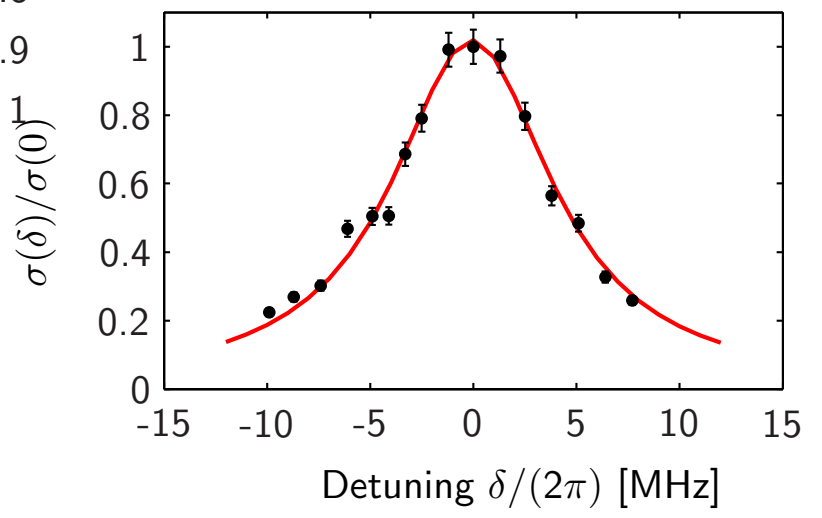

Figure 6 Optical density of a cold atom cloud in a magnetooptical trap. The probe beam is detuned from the resonance line by an amount $\delta$.

Because the two pump sources are extremely narrow in frequency, one can expect similar spectral purity of the output. At present times, we have no means to measure such narrow linewidths, but we can place an upper bound on the laser linewidth from high-resolution spectroscopy measurements.

To this aim, we used cold atoms from a magnetooptical trap (MOT) formed using the SFG laser source. Sodium atoms were introduced in a high-vacuum cell using electrically-controlled dispenser sources (Alvatec $\mathrm{GmbH}$ ). Repumping light was derived from the main laser using a high-frequency $(1.7 \mathrm{GHz})$ acousto-optical modulator (Brimrose Corp.). A MOT was formed in the vacuum cell using a magnetic field gradient around $10 \mathrm{G} / \mathrm{cm}$ and approximately $10 \mathrm{~mW}$ optical power in each of the six MOT beams. The cloud typically contained a few $10^{7}$ atoms, at a temperature $T \sim 110 \mu \mathrm{K}$. We measured the absorption of a weak probe beam (intensity $\sim 1 \mathrm{~mW} / \mathrm{cm}^{2}$ ) by the atomic cloud (with MOT beams turned off) as a function of the probe frequency. According to Beer-Lambert's law, this measures $\sigma(\delta)$, the optical density at a detuning $\delta=\omega_{L}-\omega_{0}$, with $\omega_{L}$ the laser frequency and $\omega_{0}$ the atomic resonance frequency. Typical results are plotted in Fig. 6] These mea- surements have been fitted using the theoretical expression

$$
\sigma(\delta) / \sigma(0)=\frac{\Gamma^{2} / 4}{\delta^{2}+\Gamma^{2} / 4},
$$

where $\Gamma$ is the natural linewidth for the $D_{2}$ transition of sodium. We deduce a measured value $\Gamma /(2 \pi) \approx 9.6 \pm$ $0.5 \mathrm{MHz}$, compatible with the value found in the literature, $\Gamma /(2 \pi)=9.8 \mathrm{MHz}$. Since no broadening of the absorption profile due to the linewidth of the laser could be observed within our measurement accuracy, the latter is small compared to the natural linewidth of the atoms. We conclude that the laser source fullfils all the requirements for laser cooling applications.

\section{Conclusion}

In conclusion, we have demonstrated a single-frequency, tunable, compact and robust all-solid-state SFG yellow laser source for cooling and trapping sodium atoms. The long-term stability of the laser source, despite the complexity brought by the use of a doubly-resonant enhancement cavity, stems from an original electronic servo loop. This servo is designed both to bypass the large depletion dip observed on the weaker input laser resonance fringe under high conversion, and to avoid saturation due to disturbances of the various integrators used in the servo loops. In the current configuration, the maximum output power of $800 \mathrm{~mW}$ remains lower than what can be produced with a dye laser. However, based on our measurements, we calculate that increasing the powers of the infrared laser sources to $P_{1}=2 \mathrm{~W}$ and $P_{2}=800 \mathrm{~mW}$ (both commercially available) should allow output powers in excess of $1 \mathrm{~W}$, ultimately limited by the possible occurrence of thermal effects in the crystal [29, 34, 35].

Acknowledgements We would like to thank Pierre Lemonde, Wolfgang Ketterle and Aviv Keshet for useful discussions. We acknowledge financial support from ANR (contract Gascor), IFRAF (Microbec project), the European Union (MIDAS project, Marie Curie Fellowship) and DARPA (OLE project).

\section{References}

1. K. B. Davis, M. O. Mewes, M. R. Andrews, N. J. van Druten, D. S. Durfee, D. M. Kurn, and W. Ketterle, Phys. Rev. Lett. 75, 3969 (1995).

2. L. Vestergaard Hau, B. D. Busch, C. Liu, Z. Dutton, M. M. Burns, and J. A. Golovchenko, Phys. Rev. A 58, R54 (1998).

3. D. S. Naik and C. Raman, Phys. Rev. A 71, 033617 (2005).

4. K. M. F. Magalhaes, S. R. Muniz, E. A. L. Henn, R. R. Silva, L. G. Marcassa, and V. S. Bagnato, Laser Physics Letters 2, 214 (2005). 
5. E. Streed, A. Chikkatur, T. Gustavson, M. Boyd, Y. Torii, D. Schneble, G. Campbell, D. Pritchard, and W. Ketterle, Rev. Sci. Instrum. 77, 023106 (2006).

6. R. Dumke, M. Johanning, E. Gomez, J. D. Weinstein, K. M. Jones, and P. D. Lett, New Journal of Physics 8, 64 (2006).

7. K. M. R. van der Stam, E. D. van Ooijen, R. Meppelink, J. M. Vogels, and P. van der Straten, Review of Scientific Instruments 78, 013102 (2007).

8. Z. Hadzibabic, S. Gupta, C. Stan, C. Schunck, M. Zwierlein, K. Dieckmann, and W. Ketterle, Phys. Rev. Lett. 91, 160401 (2003).

9. T. H. Jeys, A. A. Brailove, and A. Mooradian, Appl. Opt. 28, 2588 (1989).

10. H. Moosmüller and J. D. Vance, Opt. Lett. 22, 1135 (1997).

11. J. D. Vance, C. Y. She, and H. Moosmüller, Appl. Opt. 37, 4891 (1998).

12. J. C. Bienfang, C. A. Denman, B. W. Grime, P. D. Hillman, G. T. Moore, and J. M. Telle, Opt. Lett. 28, 2219 (2003).

13. J. Janousek, S. Johansson, P. Tidemand-Lichtenberg, S. Wang, J. Mortensen, P. Buchhave, and F. Laurell, Opt. Express 13, 1188 (2005).

14. Y. Feng, S. Huang, A. Shirakawa, and K.-I. Ueda, Jpn. J. Appl. Phys. 43, L722 (2004).

15. R. Mildren, M. Convery, H. Pask, J. Piper, and T. Mckay, Opt. Express 12, 785 (2004).

16. D. Georgiev, V. P. Gapontsev, A. G. Dronov, M. Y. Vyatkin, A. B. Rulkov, S. V. Popov, and J. R. Taylor, Opt. Express 13, 6772 (2006).

17. S. Sinha, C. Langrock, M. J. Digonnet, M. M. Fejer, and R. L. Byer, Opt. Lett. 31, 347 (2006).

18. R. Q. Fugate, Nature (London) 353, 144 (1991).

19. T. Nishikawa, A. Ozawa, Y. Nishida, M. Asobe, F.-L. Hong, and T. W. Hänsch, Opt. Express 17, 17792 (2009).

20. E. Mimoun, L. De Sarlo, J.-J. Zondy, J. Dalibard, and F. Gerbier, Opt. Express 16, 18684 (2008).

21. L. E. Myers, R. Eckardt, M. M. Fejer, W. R. Bosenberg, and J. W. Pierce, J. Opt. Soc. Am. B 12, 2102 (1995).

22. R. W. Boyd, Nonlinear optics (Academic Press, ADDRESS, 2003).

23. K. Fradkin, A. Arie, A. Skliar, and G. Rosenman, Applied Physics Letters 74, 914 (1999).

24. S. Emanueli and A. Arie, Applied Physics 42, 6661 (2003).

25. G. D. Boyd and D. A. Kleinman, J. Appl. Phys. 39, 3597 (1968).

26. J.-J. Zondy, D. Touahri, and O. Acef, J. Opt. Soc. Am. B 14, 2481 (1997).

27. A. Arie, G. Rosenman, V. Mahal, A. Skliar, M. Oron, M. Katz, and D. Eger, Optics Communications 142, 265 (1997).

28. S. V. Popov, S. V. Chernikov, and J. R. Taylor, Optics Communications 174, 231 (2000).

29. R. L. Targat, J.-J. Zondy, and P. Lemonde, Optics Communication 247, 471 (2005).

30. A. E. Siegman, Lasers (University Science Books, ADDRESS, 1986).

31. Y. Kaneda and S. Kubota, Appl. Opt. 36, 7766 (1997).

32. E. Mimoun, F. Gerbier, J.-J. Zondy, and J. Dalibard, French patent INPI0803153, International patent pending (2008).
33. R. W. Fox, C. W. Oates, and L. W. Hollberg, Experimental Methods in the Physical Sciences 40, (01).

34. F. Torabi-Goudarzi and E. Riis, Optics Communications 227, 389 (2003).

35. J. H. Lundeman, O. B. Jensen, P. E. Andersen, S. Andersson-Engels, B. Sumpf, G. Erbert, and P. M. Petersen, Opt. Express 16, 2486 (2008). 